\title{
Article \\ Reliability and Validity in Cranial Osteopathy Experience: A Cross-Sectional Study
}

\author{
Jesús Requena García ${ }^{1}$, Evelyn García Nieto², David Varillas Delgado³. \\ ${ }^{1}$ Formación Belga-Española de Osteopatía (FBEO), Madrid, Spain. \\ 2 Universidad Politécnica de Madrid (UPM), Higher Technical School of Industrial Engineers, Madrid, Spain. \\ ${ }^{3}$ Universidad Francisco de Vitoria, Faculty of Medicine, Research Unit, Madrid, Spain. \\ * Correspondence: David Varillas Delgado. Universidad Francisco de Vitoria (UFV), Faculty of Medicine, \\ Research Unit, Madrid, Spain., Crta. Pozuelo de Alarcón-Majadahonda Km 1,800 P.C. 28223; E-Mail: \\ david.varillas@ufv.es
}

\begin{abstract}
Background and objectives: the techniques directed to the cranial field in osteopathy are the most questioned due to the lack of scientific evidence. In osteopathic practice, manual palpation is essential and, therefore, measuring reliability is fundamental. The objective of study is to assess the reliability and validity of osteopathic treatment depending on experience. Materials and Methods: A cross-sectional study of reliability and validity was conducted. For measurements, a strain gauge was placed on the sphenobasilar synchondrosis of the skull base, and three maneuvers (lateral compression, anteroposterior compression and compression maneuver of the mastoids) were repeated 25 times each by osteopaths with different time of experience (5-10 years, 1-5 years, $<1$ year). Measurement averages were computed for each of the three maneuvers to verify the average effect of each group in comparison to that of the Gold Standard (GS) (>10 years of experience). Data were analyzed to check for inter- and intra-observer reliability using intra-class correlation coefficients (ICC). Results: Reliability and validity in 5-10 experience of observer 1 and observer 2 in the tree maneuvers was excellent $(\mathrm{p}<0.001)$ against GS. Poor or enough reproducibility and concordance were observed in osteopaths with less experience. Conclusions: Experience of osteopaths determines the efficacy of cranial maneuvers in osteopathic treatment for patients' rehabilitation.
\end{abstract}

Keywords: Osteopathic Manipulation; Cranial Osteopathy; Reproducibility; Osteopathic Medicine

\section{Introduction}

Osteopathy as a discipline was founded in the USA in 1874 by Andrew Taylor Still ${ }^{1}$. For the World Health Organization (WHO) osteopathy relies on manual contact for diagnosis and treatment, replacing the definition initially proposed by the World Osteopathic Health Organization. There exists a large heterogeneity in recognition and regulation of the practice of osteopathy across different countries, sometimes depending on whether practitioners are admitted to the medical community or not ${ }^{2,3}$. This increasing interest in osteopathic medicine may reflect the reality of the society as patients turn to complementary or alternative treatments when conventional treatments seem to fail in producing the desired outcome, or produce side effects 4,5. Among the available Osteopathic Manipulative Treatment (OMT) techniques, those addressed to the cranial field are the most questioned because of the lack of scientific evidence. A systematic review of 7 randomized controlled trials revealed that previous studies showed poor methodology and insufficient data to draw a conclusion about Osteopathy in the Cranial Field (OCF). One of the keystones of OCF teaching and practice is the presence of Primary Respiratory Mechanism (PRM), which is partially represented by the movement of the cranial bones ${ }^{6}$. Thus, hands ability to perceive PRM through the obliterated sutures of the skull in adults remains debatable, besides the lack of evidence to support this 7,8 . 
According to the original model of cranial osteopathy, intrinsic rhythmic movements of ${ }^{8}$ the human brain cause rhythmic fluctuations of cerebrospinal fluid and specific relational changes among dural membranes, cranial bones, and the sacrum ${ }^{9}$. Practitioners believe that they can palpably modify parameters of this mechanism to a patient's health advantage 9 .

A variety of statistical methods were used in early reliability experiments in manipulative medicine ${ }^{10-12}$. The current standard of reliability analysis is the $\kappa$ statistic, which has gained widespread acceptance as a measure of reliability because it accounts for the role of randomness in dichotomous agreement ${ }^{11,12}$. To date, three reviews of the literature (two of them systematic) have examined the intra and interobserver reliability of diagnostic procedures used in cranial osteopathy $8,13,14$. Osteopathic practitioners rely on the relationship between structure and function to optimize the body's self-regulating and self-healing capabilities. This holistic approach for patient care and healing is based on the concept that human beings are dynamic functional units, in which all parts are interrelated and possess self-regulatory and self-healing mechanisms. Two essential components of osteopathic health care are the structural evaluation of the patient for diagnosis and an array of manipulative techniques for treatment ${ }^{15}$. Therefore, the reliability and validity of different measuring methods are important for osteopathic efficiency.

The aim of this study was to assess the reliability and validity of osteopathic treatment based on experience.

\section{Methods}

\subsection{Study design}

Cross-sectional study of reliability and validity.

\subsection{Setting}

The measurements of the presented manoeuvres were carried out in November 2019 in the Belgian-Spanish Osteopathy Formation (Formación Belga-Española de Osteopatía; FBEO), Madrid.

\section{- Participants}

Professional osteopaths of FBEO were selected based on their experience. The first author of the study was selected as the Gold Standard (GS) for having more than 15 years of experience in cranial osteopathy. Two osteopaths (observer 1 and observer 2) with 5-10 years of experience, 2 osteopaths with 1-5 years of experience, and 2 osteopaths with less than 1 year of experience were selected to carry out the maneuvers and assess inter-observer and intra-observer reliability. Osteopaths from FBEO were sorted out based on their experience, and some of them were randomly selected to conduct our study. All the selected osteopaths agreed on taking part in the study. The age range of the participants was 23-43 years old.

All osteopaths gave their informed consent for inclusion before they participated in the study. The study was conducted in accordance with the Declaration of Helsinki, and the protocol was approved by the Ethics Committee of Universidad Francisco de Vitoria (UFV2019-33).

\subsection{Maneuver measurements}

The strain gauge with a rectangular shape is placed parallel to the sphenobasilar synchondrosis as shown in Fig. 1. 

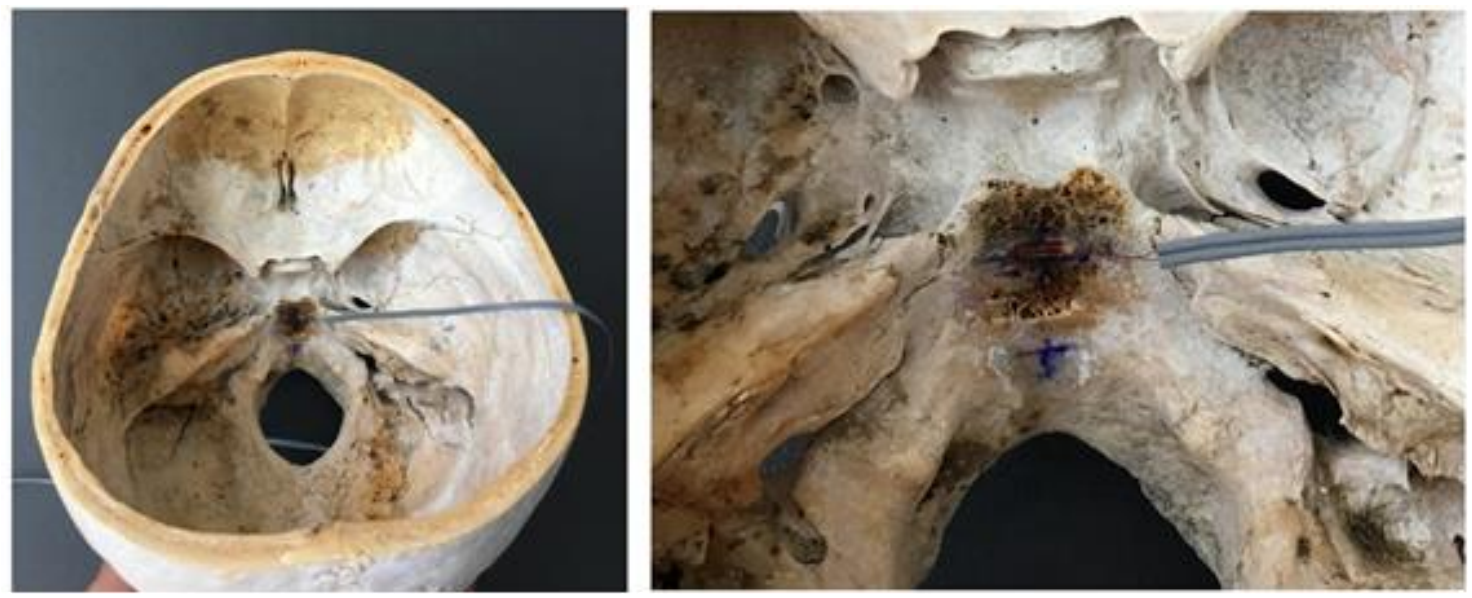

Figure 1. Location of the gauge at the base of the skull.

Three different maneuvers were repeated 25 times each by osteopaths bringing the maximum load to that point and trying to apply the same load each time. The three maneuvers selected were:

1. Lateral compression (maneuver 1): The osteopath places one hand on the occipital and the other hand on the major wings of the sphenoid. The osteopath performs an internal pressure on both the major and occipital wings (Fig. 2a).

2. Anteroposterior compression (maneuver 2): Osteopath's hands are placed on the occipital and on the frontal bone. The maneuver consists of performing an integer-posterior pressure producing a compression of the sphenobasilar (Fig. 2b).

3. Compression of mastoids (maneuver 3): The osteopath places both thumbs and thenar eminences on the mastoid processes. The action consists of pressing both mastoids in the direction of the sphenobasilar synchondrosis (Fig. 2c).
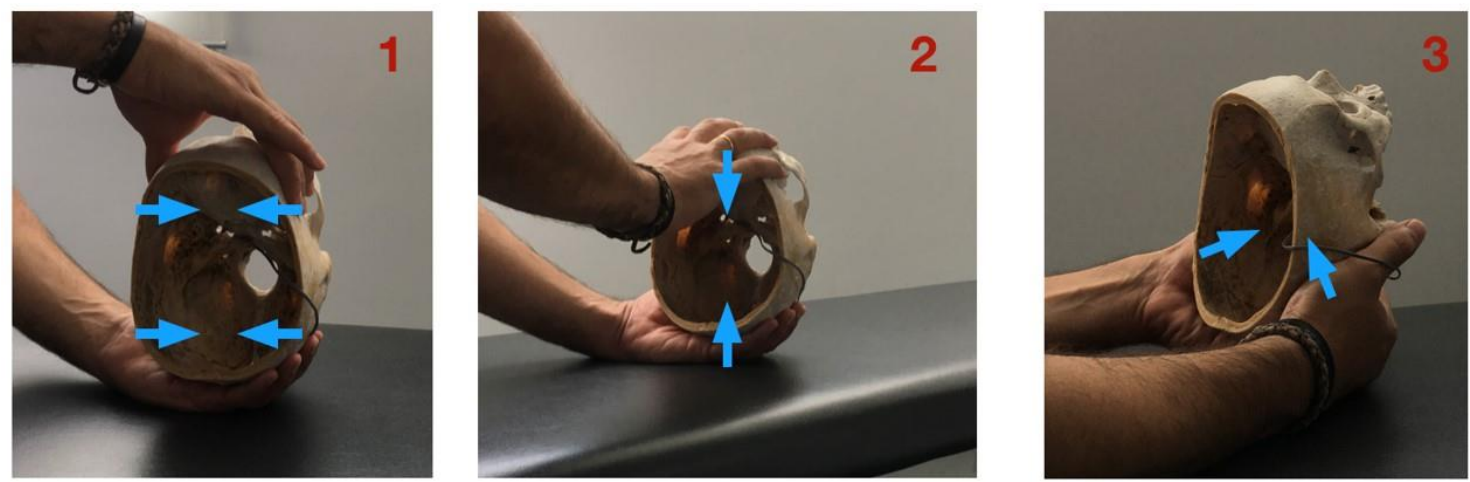

Figure 2. Manoeuvers measurements; 1 Lateral compression manoeuver, 2 Anteroposterior compression manoeuver and 3 Compression manoeuver of the mastoids.

All the information about the manoeuvre-induced deformations was registered using the MM01_multidaq software (Micro-Measurements, Vishay Precision Group, Inc. (VPG), 3 Great Valley Parkway, Malvern, Pennsylvania, E.E.U.U.), providing values in micrometres $(\mu \mathrm{m})$. Values indicate the micrometers of deformation. Positive values indicate stretching, whereas negative values indicate compression.

To minimize possible biases in the measurements, 25 repetitions in each maneuver were carried out by osteopaths. 


\subsection{Statistical analysis}

Statistical analyses were performed in SPSS 21.0® for Windows (IBM Corp. Released 2012. IBM SPSS Statistics for Windows, Version 21.0. Armonk, NY: IBM Corp, Chicago, Illinois, E.E.U.U.).

Shapiro-Wilk tests were carried out before the main statistical analyses to check for normal distribution of study variables. The mean and standard deviation (SD) were used for descriptive purposes.

Reliability was assessed with the intra-class correlation coefficient (ICC) by aleatory effects. The magnitude of concordance was classified as follows: $<0.40$ as poor, $0.40-0.59$ as enough, $0.60-0.74$ as good, and 0.75-1 as excellent.

Reliability was assessed with the Cronbach's alpha coefficient. Values equal or higher than 0.70 were considered adequate.

Level of significance for statistical tests was set at $5 \%$.

\section{Results}

Data of measurements made in the three maneuvers by osteopaths are shown in table 1.

Table 1. Measurement data in micrometers $(\mu \mathrm{m})$ and standard deviation (SD) of osteopaths in the three maneuvers.

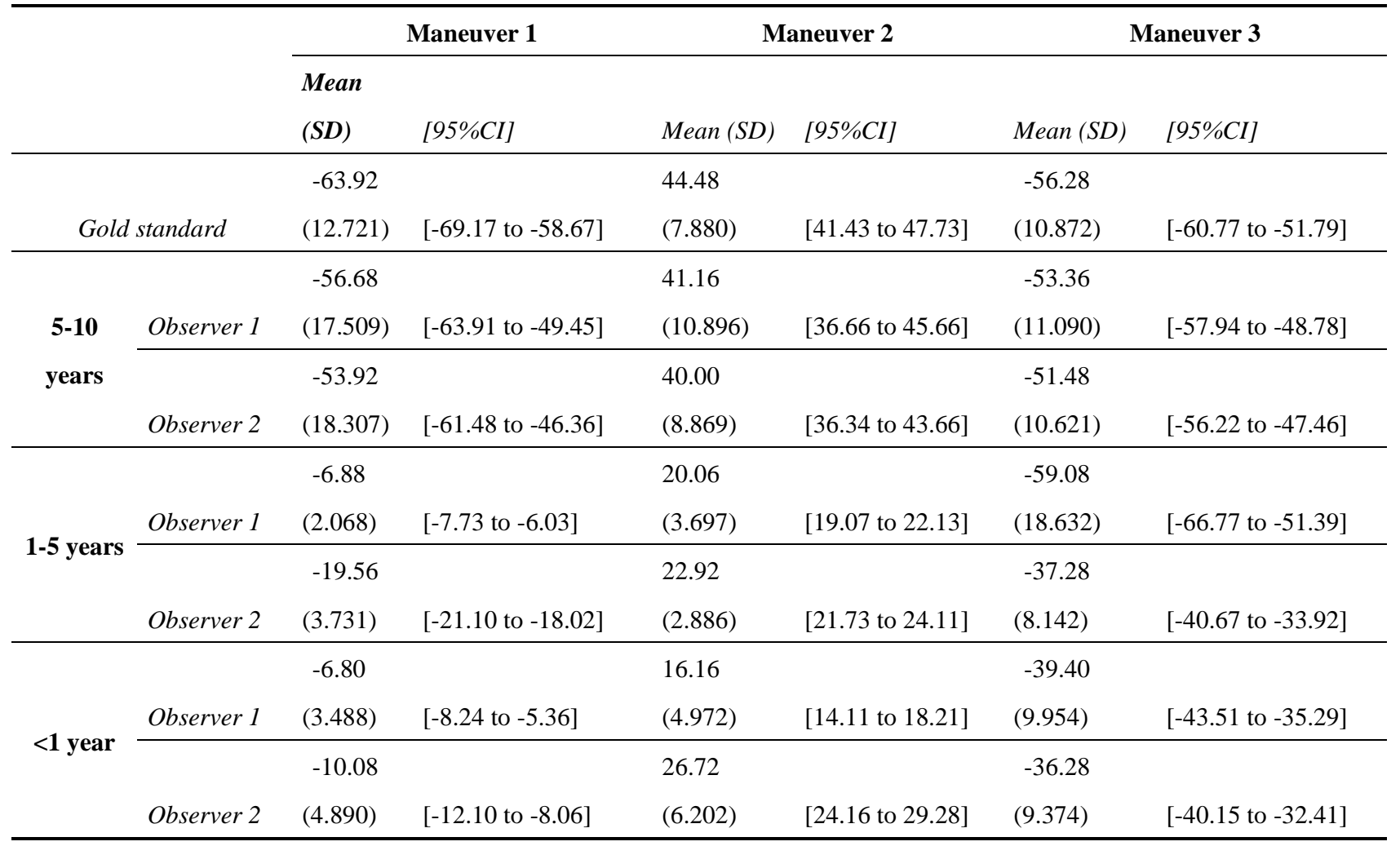

Reproducibility between GS and osteopaths with 5-10 years of experience was excellent for the tree maneuvers with ICC values of $0.809-0.861$ and $0.698-0.824$ for observers 1 and 2, respectively (ICC: $\mathrm{p}<0.001$ ). In maneuver 2, poor reproducibility and low concordance were observed in both observers with 1-5 years of experience (observer $10.015(\mathrm{p}=0.783)$ and observer $20.036(\mathrm{p}=0.683)$, but in observer 2 with less than one year of experience, concordance was confirmed close to adequate (Cronbach's alpha 0.665), but with poor reproducibility (ICC 0.216; $\mathrm{p}=0.743$ ). However, in the observers with less than one year of experience, a concordance in maneuver 3 with respect to GS was 
adequate (observer $10.752 ; \mathrm{p}=0.002$ and observer $20.841 ; \mathrm{p}<0.001$ ), but poor reproducibility through the ICC respecting gold standard (Table 2 ).

Table 2. Reproducibility analysis and concordance between the gold standard and different observers based in the experience for the action of all maneuvers.

\begin{tabular}{|c|c|c|c|c|c|c|}
\hline & & \multicolumn{5}{|c|}{ Maneuver 1} \\
\hline & & Measures & Difference mean (SD) & $95 \%$ limits of agreement & Alpha & ICC \\
\hline \multirow{2}{*}{ 5-10 years } & Observer $1-G S$ & 25 & $-7.240(10.868)$ & -11.726 to -2.754 & 0.856 & 0.809 \\
\hline & Observer $2-G S$ & 25 & $-10.000(13.491)$ & -15.569 to -4.431 & 0.776 & 0.696 \\
\hline \multirow{2}{*}{$1-5$ years } & Observer $1-G S$ & 25 & $-57.040(13.843)$ & -62.754 to -51.326 & 0.363 & 0.015 \\
\hline & Observer 2 - GS & 25 & $-44.360(11.676)$ & -49.180 to -39.540 & 0.366 & 0.036 \\
\hline \multirow{2}{*}{$<1$ year } & Observer $1-G S$ & 25 & $-57.120(10.635)$ & -61.510 to -52.730 & 0.518 & 0.035 \\
\hline & Observer 2 - GS & 25 & $-53.840(10.656))$ & -58.239 to -49.441 & 0.560 & 0.046 \\
\hline
\end{tabular}

\section{Maneuver 2}

Measures Difference mean (SD) $95 \%$ limits of agreement Alpha ICC

\begin{tabular}{|c|c|c|c|c|c|c|}
\hline 5-10 years & Observer $1-G S$ & 25 & $3.320(6.310)$ & 0.716 to 5.924 & 0.876 & 0.851 \\
\hline & Observer 2 - GS & 25 & $4.480(6.404)$ & 1.837 to 7.123 & 0.829 & 0.770 \\
\hline \multirow[t]{2}{*}{$1-5$ years } & Observer $1-G S$ & 25 & $23.880(7.965)$ & 20.592 to 27.168 & 0.280 & 0.038 \\
\hline & Observer 2 - GS & 25 & $21.560(7.969)$ & 18.271 to 24.849 & 0.179 & 0.026 \\
\hline \multirow[t]{2}{*}{$<1$ year } & Observer $1-G S$ & 25 & $28.320(9.371)$ & 24.452 to 32.188 & 0.023 & 0.002 \\
\hline & Observer 2 - GS & 25 & $17.760(7.102)$ & 14.828 to 20.692 & 0.665 & 0.216 \\
\hline
\end{tabular}

\section{Maneuver 3}

Measures Difference mean (SD) $95 \%$ limits of agreement Alpha ICC

5-10 years Observer $1-G S \quad 25 \quad-2.920(7.354) \quad-5.955$ to $0.115 \quad 0.874 \quad 0.861$




\begin{tabular}{|c|c|c|c|c|c|c|}
\hline & Observer 2 - GS & 25 & $-4.440(7.534)$ & -7.550 to -1.330 & 0.860 & 0.824 \\
\hline \multirow[t]{2}{*}{$1-5$ years } & Observer $1-G S$ & 25 & $2.800(12.261)$ & -2.261 to 7.861 & 0.807 & 0.806 \\
\hline & Observer 2 - GS & 25 & $-19.000(7.269)$ & -22.000 to -16.000 & 0.833 & 0.390 \\
\hline \multirow[t]{2}{*}{$<1$ year } & Observer $1-G S$ & 25 & $-16.880(9.293)$ & -20.716 to -13.044 & 0.752 & 0.416 \\
\hline & Observer 2 - GS & 25 & $-20.000(7.522)$ & -23.105 to -16.895 & 0.841 & 0.397 \\
\hline
\end{tabular}

The measurement performed among the observers with 5-10 years of experience, 1-5 years and $<1$ year in the three maneuvers were carried out to verify the average effect of both in the three maneuvers analyzed against the GS (Table 3), verifying that the reproducibility in the average of the evaluations of the osteopaths with 5-10 years of experience were excellent by the ICC $(0.760-0.856)$ in the three maneuvers, as well as concordance measured by Cronbach's alpha (0.826-0.899). Poor reproducibility was demonstrated in the means of the valuations of observers with less experience.

Table 3. Reproducibility analysis and concordance in the means of the observations in the three maneuvers.

\begin{tabular}{|c|c|c|c|c|c|c|c|}
\hline & & \multicolumn{2}{|c|}{ Maneuver 1} & \multicolumn{2}{|c|}{ Maneuver 2} & \multicolumn{2}{|c|}{ Maneuver 3} \\
\hline & & Alpha & ICC & Alpha & $I C C$ & Alpha & ICC \\
\hline 5-10 years & Observers - GS & 0.826 & 0.760 & 0.899 & 0.856 & 0.881 & 0.856 \\
\hline $1-5$ years & Observers - GS & 0.051 & 0.003 & 0.245 & 0.032 & 0.565 & 0.480 \\
\hline$<1$ year & Observers - GS & 0.550 & 0.040 & 0.462 & 0.077 & 0.830 & 0.413 \\
\hline
\end{tabular}

\section{Discussion}

Cranial osteopathy (CO), since its inception with Sutherland ${ }^{16}$, has demonstrated its efficacy in different pathologies and dysfunctions. In a recent systematic review 17 it is reported that osteopathic manipulation is effective against headache as a result of the manipulation of the cranial bones and the covering fasciae. CO is important not only at the symptomatology level, but also at the cost level, since headaches alone cost US \$ 36 billion annually and cause 113 million sick leave ${ }^{18}$.

The study by Haller ${ }^{19}$ showed that $\mathrm{CO}$ is effective and safe to reduce neck pain intensity, ameliorate functional disability, and improve life quality. In another systematic review on the effects of $\mathrm{CO}^{20}$ it is reported that, although the results of such a review are heterogeneous and insufficient, the effectiveness of the $\mathrm{CO}$ is well documented, showing positive results in most reviewed studies, which confirms clinical benefits.

Although the effectiveness of $\mathrm{CO}$ has been demonstrated in various fields, further research is needed regarding action means, and how to improve the education of students and the accuracy of manipulations. That is the rationale of our study.

One of the important parts that has generated controversy and is relevant in this study is to check whether structural changes occur in the manipulation of the skull. Structural changes have 
been demonstrated in different studies, such as that of Kostopoulos and Keramidas ${ }^{21}$, which provides scientific evidence that forces applied to the skull through $\mathrm{CO}$ techniques produce an elongation of the brain sickle. In their study, authors use an embalmed corpse in which cranial membranes had become harder and more resistant to movement. Another study demonstrating structural changes 22 concluded that cranial bone mobility can be documented and measured with X-rays, data that has not been showed in this work, but that encourages us to carry out subsequent studies to confirm these findings.

In addition to demonstrating structural changes, further studies have been carried out on the mechanical load needed to produce such changes. The study by Downey et al. ${ }^{23}$ simulated a craniosacral treatment technique: (frontal lifting through applying precisely measured distraction forces) and a change in intracranial pressure and movement through the coronal suture were observed in an animal after the application of forces greater than those used clinically in the practice of $\mathrm{CO}$, obtaining results with traction of 1000 gr. Another study in the same direction is the review about cranial motion by Seimetz et al ${ }^{24}$, where it is illustrated that both externally applied forces and increased intracranial pressure result in measurable movement through cranial sutures in young and adult mammals, and measurable changes in the diameter of the cranial vault in human skulls of living and post-mortem adults. However, the magnitude of cranial movement may vary depending on the subject, the head region where forces are applied, and the method of force application. This last conclusion of the study by Seimetz et al. ${ }^{24}$ highlights the importance of the different methods to apply forces in the skull, which agrees with the conclusion of our study.

The great pending subject of $\mathrm{CO}$, which also motivates this study, is diagnostic evaluation. There are studies that show differences in tactile ability between different students, such as the study by Nascimiento et al. ${ }^{25}$, which states that teaching strategies used during different educational periods can contribute to improve tactile sensitivity and precision of manual palpation in health sciences students. In this respect, another study investigated the effects of standardized protocol training on cranial palpation pressures used by osteopathy students 26 , and concluded that palpatory training was ineffective in improving accuracy in students of cranial palpation, also suggesting to examine palpation pressures used by experienced professionals. Both studies ${ }^{25,26}$ ultimately highlighted the importance of training in student palpation, a conclusion shared with the results of our study.

A study assessing the inter and intra-observer reliability in palpation of the primary respiratory mechanism within the cranial concept was carried out by Sommerfeld et al. ${ }^{8}$. Their results showed that PRM could not be reliably palpated and, under certain conditions, was influenced by the respiratory rates of examiners. These results do not support the hypotheses based on the role of PRM palpation for clinical decision making.

Currently, the skull can be considered a model of tensegrity and functional unity as stated in Scarr ${ }^{27}$, where it is concluded that tension forces in dura mater have the effect of separating bones, as well as integrating them into a single functional unit. This alteration of the duramater or suture occurs in a specific anatomical area of the skull. Therefore, it is important for the reliability of both diagnosis and treatment that osteopaths know exactly to which anatomical area his palpation corresponds.

If the treatment approach is assessed, a scientific explanation can be found in the mechanotransduction 28 , mechanisms by which cells convert mechanical stimuli into cellular responses, which brings back to terms the importance of treatment accuracy to exert mechanical loads on the right place. Data from this study give even more importance to the fact that manoeuvres should be precise as much as possible.

Other issue to be solvedwas whether hands can perceive movements and minimal changes that occur in the cranial tissue. In this regard, the study by Kasparian et al. ${ }^{28}$ determined that one third of our samples was capable to detect movements of less than $50 \mu \mathrm{m}$.

Thus, the authors of this article share the previously stated idea of why not treating the skull like any other body structure ${ }^{29}$.

Our study can contribute to scientific progress in this area, making a precision model of reliability, evaluation and education. As described before, $\mathrm{CO}$ has a lot of beneficial effects in humans 
with a scientific and physiological explanation through mechano-transduction. However, there is no model that explains where and how to perform manoeuvres or mechanical loads to act in a specific anatomical area of the skull. Thus, this model can indicate potential differences between different osteopaths.

The results obtained in this study show that cranial osteopathy depends not only on sensitivity, but can be also trained, practiced and improved objectively. There are differences between measurements of osteopaths with different years of experience, which shows that experience is very important for possible clinical outcomes.

The limitations of this study are the choice of a small group of osteopaths from a Spanish school, so the results cannot be extrapolated to the entire osteopath community in our country and be representative of it, but they provide knowledge about the methodology and offers an area of improvement in the teaching of cranial osteopathy. These results open new way of knowledge should be carried out in subsequent studies to corroborate these results.

\section{Conclusions}

We confirmed a higher reliability and validity in osteopaths with more years of work in comparison to those with fewer years in the efficacy of cranial maneuvers. The presented data open a new possibility in the treatment efficacy through maneuvers of cranial osteopathy used for patient rehabilitation.

\section{Ethical statement}

All osteopaths gave their informed consent for inclusion before they participated in the study. The study was conducted in accordance with the Declaration of Helsinki, and the protocol was approved by the Ethics Committee of Universidad Francisco de Vitoria (UFV2019-33).

Acknowledgments: To the Belgian-Spanish Osteopathy Formation (Formación Belga-Española de Osteopatía FBEO) for transferring the facilities and the material to carry out the study measurements and to the collaborating osteopaths for carried out the different maneuvers.

Author Contributions: Conceptualization, J.R.G. and D.V.D.; Methodology, E.G.N. and D.V.D..; Software, E.G.N.; Validation, J.R.G., E.G.N. and D.V.D..; Formal Analysis, D.V.D.; Investigation, J.R.G. and D.V.D.; Writing - Original Draft Preparation, J.R.G. and D.V.D.; Writing - Review \& Editing, J.R.G, E.G.N. and D.V.D..; Visualization, J.R.G.; Supervision, D.V.D.

Conflicts of Interest: The authors declare no conflict of interest.

\section{References}

1. (1) Still, G. F. On a Form of Chronic Joint Disease in Children. Med Chir Trans 1897, 80, 47.

2. (2) Luciani, E.; Consorti, G.; van Dun, P. L. S.; Merdy, O.; Lunghi, C.; Petracca, M.; Esteves, J. E.; Cerritelli, F. An overview of osteopathy graduates' perceived preparedness at transition from educational environment to clinic environment one year after graduation: a cross sectional study. BMC Med Educ 2018, 18 (1), 319.

3. (3) Guillaud, A.; Darbois, N.; Monvoisin, R.; Pinsault, N. Reliability of Diagnosis and Clinical Efficacy of Cranial Osteopathy: A Systematic Review. PLoS One 2016, 11 (12), e0167823.

4. (4) Dossett, M. L.; Cohen, E. M.; Cohen, J. Integrative Medicine for Gastrointestinal Disease. Prim Care 2017, 44 (2), 265.

5. (5) Martins, W. R.; Diniz, L. R.; Blasczyk, J. C.; Lagoa, K. F.; Thomaz, S.; Rodrigues, M. E.; de Oliveira, R. J.; Bonini-Rocha, A. C. Immediate changes in electroencephalography activity in individuals with nonspecific chronic low back pain after cranial osteopathic manipulative treatment: study protocol of a randomized, controlled crossover trial. BMC Complement Altern Med 2015, 15, 223.

6. (6) Rosen, M. E. In J Am Osteopath Assoc United States, 2009; Vol. 109.

7. (7) Sabini, R. C.; Elkowitz, D. E. Significance of differences in patency among cranial sutures. J Am Osteopath Assoc 2006, 106 (10), 600. 
8. (8) Sommerfeld, P.; Kaider, A.; Klein, P. Inter- and intraexaminer reliability in palpation of the "primary respiratory mechanism" within the "cranial concept". Man Ther 2004, 9 (1), 22.

9. (9) Hartman, S. E. Cranial osteopathy: its fate seems clear. Chiropr Osteopat 2006, 14, 10.

10. (10) Adhia, D. B.; Bussey, M. D.; Ribeiro, D. C.; Tumilty, S.; Milosavljevic, S. Validity and reliability of palpation-digitization for non-invasive kinematic measurement - a systematic review. Man Ther 2013, 18 (1), 26.

11. (11) Haas, M. The reliability of reliability. J Manipulative Physiol Ther 1991, 14 (3), 199.

12. (12) Haas, M. Statistical methodology for reliability studies. J Manipulative Physiol Ther 1991, 14 (2), 119.

13. (13) Green, C.; Martin, C. W.; Bassett, K.; Kazanjian, A. A systematic review of craniosacral therapy: biological plausibility, assessment reliability and clinical effectiveness. Complement Ther Med 1999, 7 (4), 201.

14. (14) Moran, R. W.; Gibbons, P. Intraexaminer and interexaminer reliability for palpation of the cranial rhythmic impulse at the head and sacrum. J Manipulative Physiol Ther 2001, 24 (3), 183.

15. (15) Hayden, C.; Mullinger, B. A preliminary assessment of the impact of cranial osteopathy for the relief of infantile colic. Complement Ther Clin Pract 2006, 12 (2), 83.

16. (16) Bordoni, B.; Zanier, E. Sutherland's legacy in the new millennium: the osteopathic cranial model and modern osteopathy. Adv Mind Body Med 2015, 29 (2), 15.

17. (17) Whalen, J.; Yao, S.; Leder, A. A Short Review of the Treatment of Headaches Using Osteopathic Manipulative Treatment. Curr Pain Headache Rep 2018, 22 (12), 82.

18. (18) Khusid, M. A. Clinical indications for acupuncture in chronic post-traumatic headache management. Mil Med 2015, 180 (2), 132.

19. (19) Haller, H.; Lauche, R.; Cramer, H.; Rampp, T.; Saha, F. J.; Ostermann, T.; Dobos, G. Craniosacral Therapy for the Treatment of Chronic Neck Pain: A Randomized Sham-controlled Trial. Clin J Pain 2016, $32(5), 441$.

20. (20) Jakel, A.; von Hauenschild, P. Therapeutic effects of cranial osteopathic manipulative medicine: a systematic review. J Am Osteopath Assoc 2011, 111 (12), 685.

21. (21) Kostopoulos, D. C.; Keramidas, G. Changes in elongation of falx cerebri during craniosacral therapy techniques applied on the skull of an embalmed cadaver. Cranio 1992, 10 (1), 9.

22. (22) Oleski, S. L.; Smith, G. H.; Crow, W. T. Radiographic evidence of cranial bone mobility. Cranio 2002, $20(1), 34$.

23. (23) Downey, P. A.; Barbano, T.; Kapur-Wadhwa, R.; Sciote, J. J.; Siegel, M. I.; Mooney, M. P. Craniosacral therapy: the effects of cranial manipulation on intracranial pressure and cranial bone movement. J Orthop Sports Phys Ther 2006, 36 (11), 845.

24. (24) An investigation of cranial motion through a review of biomechanically based skull deformation literature. International Journal of Osteopathic Medicine 2012, 15 (4), 152

25. (25) Comparative assessment of tactile sensitivity between undergraduate and postgraduate health sciences students. International Journal of Osteopathic Medicine 2016, 19, 13

26. (26) Zegarra-Parodi, R.; de Chauvigny de Blot, P.; Rickards, L. D.; Renard, E. O. Cranial palpation pressures used by osteopathy students: effects of standardized protocol training. J Am Osteopath Assoc 2009, 109 (2), 79.

27. (27) Scarr, G. A model of the cranial vault as a tensegrity structure, and its significance to normal and abnormal cranial development. International Journal of Osteopathic Medicine - INT J OSTEOPATH MED 2008, 11,80 .

28. (28) Kasparian, H.; Signoret, G.; Kasparian, J. Quantification of Motion Palpation. J Am Osteopath Assoc 2015, 115 (10), 604.

29. (29) Basic-kes, V.; Basic-Jukic, N.; Kes, P.; Demarin, V.; Labar, B. [Neurologic sequelae of bone changes in multiple myeloma and its therapy]. Acta Med Croatica 2002, 56 (3), 103. 